\title{
Visual Simulation Model of Highline Cable Alongside Replenishment for Solid Cargo
}

\author{
Weicheng Lun, Qun $\mathrm{Li}^{*}$, and Hongtao Hou \\ College of Systems Engineering, National University of Defense Technology, Changsha, 410073, China
}

\begin{abstract}
Because there have been few studies on visual simulation of highline cable thus far, a simulation model that reflects how the shape of highline cable changes visually at various replenishment stages of alongside replenishment for solid cargo was proposed. With analysis of forces exerted on highline cable, expressions of several mechanical properties and parametric equations that described the shape of highline cable were deduced using the theory of catenary and structural mechanics, which comprised algorithmic sub models of different stages. Then, based on such algorithmic sub models, a series of physical sub models were established in the Unity platform. Both algorithmic sub models and physical sub models constituted the simulation model. This kind of model differed from the pure mathematical model and theoretical simulation model because it was able to not only display how the shape of highline cable changed qualitatively but also manifest values of these relevant physical properties in real time quantificationally. Results of executions indicate that this model can reliably mirror changes of highline cable at various stages of replenishment.
\end{abstract}

Keywords: replenishment at sea; highline cable; simulation; structural mechanics; visualization; catenary

(Submitted on May 20, 2018; Revised on June 29, 2018; Accepted on July 19, 2018)

(C) 2018 Totem Publisher, Inc. All rights reserved.

\section{Introduction}

Highline Cable is used mostly in alongside replenishment, which is an important method of replenishment at sea. Since stability and security of the whole replenishment system depend on the mechanical properties of Highline Cable [1], establishing a model of highline cable to research such properties is an essential field of study on replenishment at sea.

As for studies in recent years, Nan Li et al. [2-5] successively put forward the multi-rigid-body dynamics model, the rigidflexible-mixed-body dynamics model, and the multi-flexible-body dynamics model of highline cable using the multi-body dynamics theory. They also set up the parametric design platform of highline cable by ADAMS to parameterize the optimization design model of highline cable, which is based on the multi-flexible-body dynamics model. Wei Zhao et al. [6] established a mathematic model of alongside solid cargo underway replenishment system at sea based on the analysis of several control systems' performances and the simulation model via Matlab/Simulink software. However, all of these models are only capable of analyzing some mechanical properties theoretically and cannot distinguish physical, visual changes that highline cable has experienced during replenishment.

It is obvious that there is a lack of supportive academic studies and special technological tools to set up a visual model of highline cable. Hence, this paper tries to visually simulate highline cable during replenishment based on the analysis of forces exerted for highline cable at various replenishment stages. A series of simulation models are constructed using Unity software and theories of catenary and structural mechanics, which can not only display how the shape of highline cable changes but also manifest values of certain physical properties.

\footnotetext{
* Corresponding author.

E-mail address: liqun@nudt.edu.cn
} 


\section{Overview of Alongside Replenishment and Model}

Alongside replenishment means that when a replenishing vessel sails abreast at the same speed as the receiving vessel while maintaining a safe distance, cargos are delivered by highline cable (which refers to replenishment for solid cargo) or hose (which refers to replenishment for liquid cargo), as shown in Figure 1 [7]. Highline cable is a kind of wire cable with high strength and flexibility, and it meets the definition of span wire in structural mechanics.

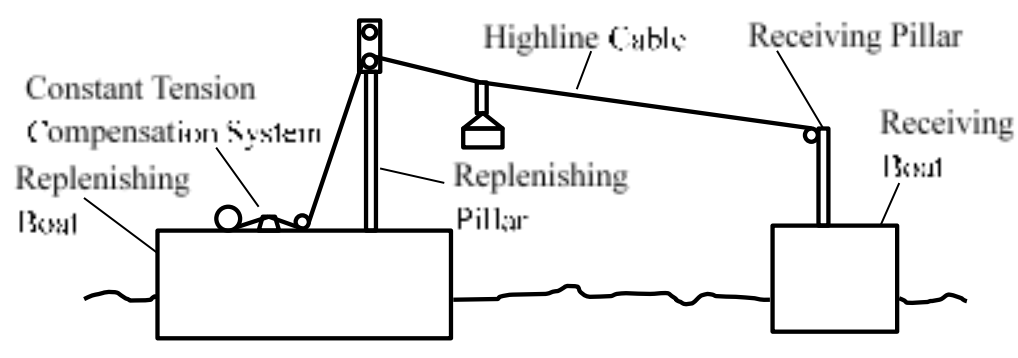

Figure 1. Schematic of alongside replenishment

Processes of replenishment can be divided into six stages: erection, stabilization, adjustment, delivery, unloading, and withdrawal. On the whole, requirements of models at all stages are similar, that is, not only manifesting values of these relevant physical properties in real time quantificationally but also displaying how shapes of highline cable change qualitatively. Meanwhile, requirements of specific stages are different.

The simulation model of the erection stage is required to display the process that highline cable elongates from replenishing vessel to receiving vessel as it is dragged by the traction cable. It must also print coordinates of junction between highline cable and traction cable as well as the instantaneous length of the highline cable on the screen. The simulation model of the withdrawal stage is required to display the inverse process, that is, the highline cable is withdrawn from the receiving vessel to the replenishing vessel together with the traction cable. It must also print the same parameters as the simulation model of the erection stage.

The simulation model of the stabilization stage is required to display the shape of highline cable under the action of its gravity after erection. As the shape equation of highline cable and tensions of sending extremity (i.e., starting point of highline cable) and receiving extremity (i.e., ending point of highline cable) all change dynamically at this stage, their values need to be printed on the screen in time. Although the length of highline cable stays constant, its value should also be displayed.

The simulation model of the adjustment stage is required to display how the shape of highline cable changes due to its length changes and print the shape equation of highline cable, its length and tension of receiving extremity, and the fixed value of tension of sending extremity. The simulation model of the unloading stage is required to display the process that cargo and receiving extremity of highline cable move down along the receiving pillar and print the same parameters as the simulation model of the adjustment stage.

The simulation model of the delivery stage is required to display the shape of highline cable under the action of gravity of cargo and how the shape of highline cable changes when the cargo moves. Because the shape equation of highline cable as well as its length and tensions of receiving extremity and sending extremity all change dynamically at this stage, their values need to be updated on the screen in time.

To meet the above requirements, the simulation model will consist of an algorithmic sub model that is used to calculate and a physical sub model that is used to display. They can invoke and affect each other: calculations of the algorithmic sub model are based on parameter input from the physical sub model, some of which are just attributes of objects in the physical sub model, while the physical sub model displays calculated results of the algorithmic sub model, and changes attribute values according to these results.

All simulation models work based on the following presumptions: 
- Highline cable has ideal flexibility, which means it only withstands axial tension and cannot undergo compression or anti-bending [8].

- The gravity of highline cable distributes vertically and uniformly along its geometry. Physical properties of any points on the highline cable are the same.

- The cross-sectional area of highline cable does not change all the time.

- Do not consider the influence of vessel motions under the force of seawater, i.e., presume that the replenishing vessel and receiving vessel remain immobile during replenishment.

\section{Algorithmic Sub Model under the Action of Distributed Load}

There are two kinds of loads that are exerted on highline cable during replenishment: distributed load and concentrated load. Highline cable is under the action of the concentrated load at the delivery stage, while it is under the action of the distributed load at all the other stages. Then, these two situations will be analyzed separately.

In what follows in this paper, point $A$ is used to represent the junction between highline cable and replenishing pillar, and point $B$ is used to represent the junction between highline cable and receiving pillar. These two points become the sending extremity and receiving extremity of highline cable respectively after it is erected. The coordinate system in these two sections is built in the plane where the highline cable is located. In this coordinate, the positive direction of the $x$-axis is the horizontal right direction and the positive direction of the $y$-axis is the vertical downward direction. The origin is point $A$. In the following sections, unless there is an especial explanation, $c$ is always applied to represent the vertical distance between the sending extremity and receiving extremity whose value is $\left|y_{A}-y_{B}\right|$, which denotes the absolute value of the difference between the $y$-coordinates of point $A$ and point $B ; l$ is always applied to represent the horizontal distance between the sending extremity and receiving extremity whose value is $\left|x_{A}-x_{B}\right|$, which denotes the absolute value of the difference between $x$-coordinates of point $A$ and point $B ; \theta$ is always applied to represent the angle between chord $A B$ and the horizontal, which means $\tan \theta=\frac{c}{l}=\frac{\left|y_{A}-y_{B}\right|}{\left|x_{A}-x_{B}\right|}$.

\subsection{Erection Stage and Withdrawal Stage}

Erection requires the highline cable to be dragged by the traction cable. At this stage, one extremity of highline cable links with one extremity of traction cable that will drag the highline cable moving from replenishing vessel to receiving vessel. There is the inverse process at the withdrawal stage. The positional relationship and shapes of highline cable (labeled using a thick line) and traction cable (labeled using a thin line) are showed in Figure 2.

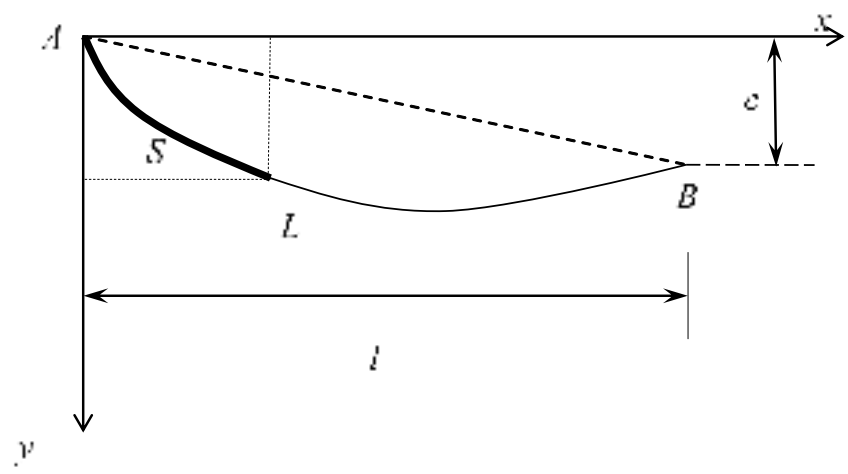

Figure 2. Schematic of erection stage and withdrawal stage

In Figure 2, $S$ denotes the instantaneous length of highline cable and point $L$ denotes the junction between highline cable and traction cable. At the erection stage, given elongate rate $v_{1}$, the instantaneous length of the highline cable can be obtained using $S=v_{1} t$ at arbitrary simulation moment $t$. Similarly, at the withdrawal stage, when the withdrawal rate is $v_{2}$, the instantaneous length of the highline cable can be calculated using $S=v_{2} t$. 


\subsection{Stabilization Stage}

The stabilization stage occurs after the highline cable is erected and before the constant tension compensation system is operative, at which positions of sending extremity and receiving extremity and the length of highline cable is fixed and the highline cable droops spontaneously under the action of distributed load $q$ which is generated from its gravity. This is shown in Figure 3.

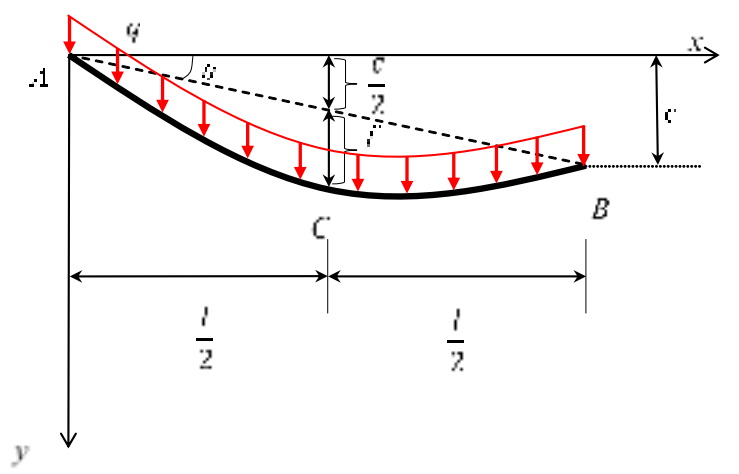

Figure 3. Schematic of stabilization stage

In Figure 3, point $C$ is the mid-span of the highline cable, which denotes that the horizontal distance between point $C$ and point $A$ equals the horizontal distance between point $C$ and point $B ; f$ is the mid-span deflection that denotes vertical distance between mid-span and chord $A B$, i.e., $f=\left|y_{A}-y_{C}\right|-\frac{c}{2} ; q$ is the distributed load along the direction of shape of highline cable.

Actually, highline cable is a kind of span wire that means an inextensible hanging wire under its own weight [9]. Its shape is a special curve called catenary. The shape equation based on catenary theory of highline cable in Figure 3 is defined as (1):

$$
y=\frac{l}{2 \beta}\left[\cosh \alpha-\cosh \left(\frac{2 \beta}{l} x-\alpha\right)\right]
$$

$\beta$ and $\alpha$ are parameters which are defined as (2) and (3) respectively [10]:

$$
\begin{gathered}
\beta=\frac{q l}{2 F_{\mathrm{H}}} \\
\alpha=\operatorname{arsinh}\left(\frac{\beta \tan \theta}{\sinh \beta}\right)+\beta
\end{gathered}
$$

$F_{\mathrm{H}}$ in (2) denotes the horizontal tension of the point on the highline cable, which is constant for any points because there is no load exerted on highline cable in the horizontal direction.

It is obvious that $\beta$ and $\alpha$ of (1) can be obtained if $c, l, q$, and $F_{\mathrm{H}}$ are known. However, $F_{\mathrm{H}}$ is just a component force of $F_{\mathrm{T}}$, i.e., the tension of every point on the highline cable, rather than an actual force. Therefore, $F_{\mathrm{H}}$ cannot be given in advance. In the ordinary way, $c, l, f$, and $q$ are known because $c, l$, and $f$ can be calculated according to the initial positions of points $A$, $B$, and $C$, and $q$ is a given constant.

The first step to compute the values of $\beta$ and $\alpha$ is to describe the coordinates of point $C$ using $c$ and $l$, that is, $y_{C}=f+c / 2$ and $x_{C}=l / 2$; then, putting them into (1) will obtain an equation represented in (4): 


$$
f+\frac{c}{2}=\frac{l}{2 \beta}[\cosh \alpha-\cosh (\beta-\alpha)]
$$

Plug (3) into (4) and $\alpha$ will be eliminated, so that (5) can be obtained with further simplification:

$$
f=\left(\tanh \frac{\beta}{2}\right) \sqrt{\frac{c^{2}}{4}+\left(\frac{l}{2 \beta} \sinh \beta\right)^{2}}
$$

Formula (5) is a transcendental equation, and its analytic solutions cannot be calculated. Since there are not more known qualities, the Newton iterative method can be applied to solve the equation represented in (5). Firstly, build the function $u(\beta)$ on the basis of (5):

$$
u(\beta)=\left(\tanh \frac{\beta}{2}\right) \sqrt{\frac{c^{2}}{4}+\left(\frac{l}{2 \beta} \sinh \beta\right)^{2}}-f
$$

Then, take the derivative of $u(\beta)$ to get $u^{\prime}(\beta)$, as (7) shows:

$$
u^{\prime}(\beta)=\frac{1}{2 \cosh ^{2} \beta} \sqrt{\frac{c^{2}}{4}+\left(\frac{l}{2 \beta} \sinh \beta\right)^{2}}+\frac{\frac{l^{2}}{4 \beta^{2}} \sinh \beta \cosh \beta-\frac{l^{2}}{4 \beta^{3}} \sinh ^{2} \beta}{\sqrt{\frac{c^{2}}{4}+\left(\frac{l}{2 \beta} \sinh \beta\right)^{2}}} \tanh \frac{\beta}{2}
$$

Iterative rules shown in (8) can be constituted, which will be applied to solve the transcendental equation represented in (5) using the Newton method, and deduce the value of $\beta$ :

$$
\left\{\begin{array}{l}
\beta_{0}=\frac{4 f}{l} \\
\beta_{k+1}=\beta_{k}-\frac{u(\beta)}{u^{\prime}(\beta)}
\end{array}\right.
$$

The initial value of $\beta$ is based on putting $F_{\mathrm{H}}=\left(q l^{2}\right) /(8 f)$ into (2). This formula is a conclusion of the parabola theory, which is an approximate solution of the catenary theory. The value of $\alpha$ can be obtained by plugging the result of the iterative into (3). Then, the shape equation of highline cable will be determined.

Once (1) is definite, its derivative is also definite, as (9) shows:

$$
\frac{\mathrm{d} y}{\mathrm{~d} x}=-\sinh \left(\frac{2 \beta}{l} x-\alpha\right)
$$

Plugging (9) into the curve arc length integral equation will obtain the length of highline cable:

$$
S=\int_{0}^{l} \sqrt{1+\left(\frac{\mathrm{d} y}{\mathrm{~d} x}\right)^{2}} \mathrm{~d} x=\sqrt{c^{2}+\left(\frac{l \sinh \beta}{\beta}\right)^{2}}
$$

On the other hand, the tension of any points on the highline cable can be calculated through (11):

$$
F_{\mathrm{T}}=F_{\mathrm{H}} \sqrt{1+\left(\frac{\mathrm{d} y}{\mathrm{~d} x}\right)^{2}}=\frac{q l}{2 \beta} \cosh \left(\frac{2 \beta}{l} x-\alpha\right)
$$

In particular, the tension of sending extremity, marked as $F_{\mathrm{TA}}$, and the tension of receiving extremity, marked as $F_{\mathrm{T} B}$, are just the special cases of (11) when $x$ equals 0 and 1 respectively, and they can be calculated using (12) and (13): 


$$
\begin{gathered}
F_{\mathrm{T} A}=\frac{q l}{2 \beta} \cosh \alpha \\
F_{\mathrm{T} B}=\frac{q l}{2 \beta} \cosh (2 \beta-\alpha)
\end{gathered}
$$

We cannot apply the length of highline cable obtained at the erection stage to infer the shape equation because part of the highline cable will be used to combine with fixtures on the receiving vessel, so that the highline cable can be erected safely. Consequently, the length of highline cable at the erection stage is usually longer than that at the stabilization stage. In reality, if the length of highline cable is known whereas mid-span deflection is unknown, another method can be applied to compute the values of $\beta$ and $\alpha$.

Firstly, set up an equation based on (10):

$$
\frac{\sinh \beta}{\beta}=\sqrt{\frac{S^{2}-c^{2}}{l^{2}}}
$$

Formula (14) is a transcendental equation that can be solved using the secant method. In order to apply the secant method, two initial values of $\beta$ are necessary. Replace $\sinh \beta$ with a power series expansion, and an inequation represented in (15) will be made:

$$
\sqrt{\frac{S^{2}-c^{2}}{l^{2}}}=\frac{1}{\beta}\left(\frac{\beta}{1 !}+\frac{\beta^{3}}{3 !}+\frac{\beta^{5}}{5 !}+\cdots\right)=1+\frac{\beta^{2}}{3 !}+\frac{\beta^{4}}{5 !}+\cdots>1+\frac{\beta^{2}}{3 !}
$$

Solving the equation represented in (15) will get:

$$
0<\beta<\sqrt{\frac{6\left(\sqrt{S^{2}-c^{2}}-l\right)}{l}}
$$

Then, one of the initial values can be obtained from (16). Meanwhile, because there is

$$
\sqrt{\frac{S^{2}-c^{2}}{l^{2}}}=\frac{\sinh \beta}{\beta}<e^{\beta}
$$

solving inequation represented in (17) will get:

$$
\beta>\ln \sqrt{\frac{S^{2}-c^{2}}{l^{2}}}
$$

At last, mix (16) and (18) to make iterative rules shown in (19), which is used to solve the equation represented in (14) via the secant method:

$$
\left\{\begin{array}{l}
\beta_{0}=\ln \sqrt{\frac{S^{2}-c^{2}}{l^{2}}} \\
\beta_{1}=\sqrt{\frac{6\left(\sqrt{S^{2}-c^{2}}-l\right)}{l}} \\
\beta_{k+1}=\beta_{k}-\frac{p\left(\beta_{k}\right)}{p\left(\beta_{k}\right)-p\left(\beta_{k-1}\right)}\left(\beta_{k}-\beta_{k-1}\right) \\
p(\beta)=\frac{\sinh \beta}{\beta}-\sqrt{\frac{S^{2}-c^{2}}{l^{2}}}
\end{array}\right.
$$




\subsection{Adjustment Stage and Unloading Stage}

The adjustment stage is the process through which the constant tension compensation system works. This system keeps the tension of the sending extremity constant with adjusting lengths of highline cable, so that deflection of highline cable will be controlled in a safe range. This means that after this system is operative, tension of sending extremity $F_{\mathrm{T} A}$ will become constant. Under these circumstances, (12) becomes an equation about $\beta$ and $\alpha$ that can be used to compute $\beta$ and $\alpha$. Formula (12) is also a transcendental equation like (5), and its analytic solutions cannot be calculated. However, it can be solved using dichotomy. Firstly, plugging (3) into (12) will eliminate $\alpha$, and then an inequation represented in (20) can be obtained with further simplification:

$$
F_{\mathrm{T} A}=\frac{q l}{2 \beta}\left[\sqrt{1+\left(\frac{\beta \tan \theta}{\sinh \beta}\right)^{2}} \cosh \beta+\beta \tan \theta\right]>\frac{q l}{2 \beta}\left[\sqrt{\left(\frac{\beta \tan \theta}{\sinh \beta}\right)^{2}} \cosh \beta+\beta \tan \theta\right]=\frac{q l \tan \theta}{2 \tanh \beta}+\frac{q l \tan \theta}{2}
$$

Solving this inequation will get:

$$
\beta>\operatorname{artanh} \frac{q l \tan \theta}{2 F_{\mathrm{TA}}-q l \tan \theta}
$$

There is $\beta_{0}=4 f / l$ in (8), which is deduced using the parabola theory. In fact, $f / l$ is also an important parameter called the central flexibility factor. A catenary whose central flexibility factor is more than 0.25 will not be suitable for the shape of span wire in the interests of safety and possibility of construction. Hence, $4 \times 0.25=1$ can be used as the upper limit. Then, an interval that is applied to solve the equation represented in (12) using dichotomy can be obtained:

$$
\operatorname{artanh} \frac{q l \tan \theta}{2 F_{\mathrm{TA}}-q l \tan \theta}<\beta<1
$$

As for the unloading stage, the force condition of highline cable is completely the same as the adjustment stage. Consequently, the same algorithm sub model can be used to deduce relevant parameters of highline cable at the unloading stage.

\section{Algorithmic Sub Model under the Action of Concentrated Load}

The concentrated load is erected at the delivery stage when the cargo moves from sending extremity to receiving extremity along the highline cable. During this process, the cargo whose gravity is far greater than the gravity of the highline cable acts as the concentrated load, which makes cable segments between the action point of the concentrated load and each extremity of highline cable approximate the straight segment. Therefore, these cable segments can be treated as straight segments according to the theory of structure mechanics [10]. 4.

Here is a highline cable that is labeled as $A B$, and it is under the action of concentrated loads $G$, which is shown in Figure

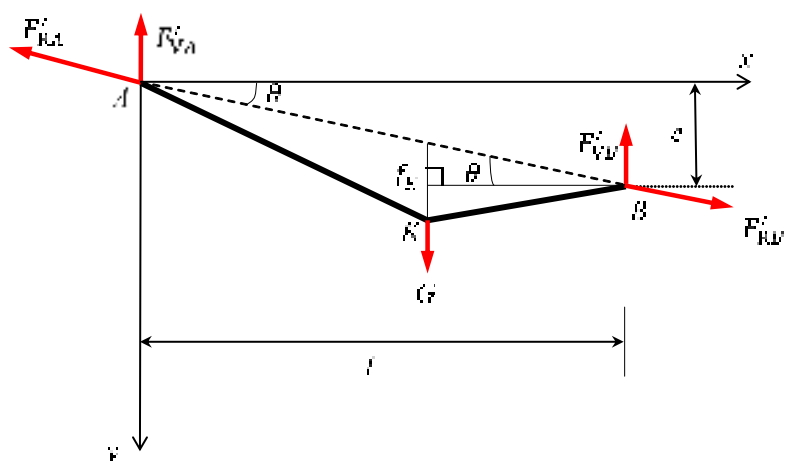

(a) Calculation schematic of concentrated load

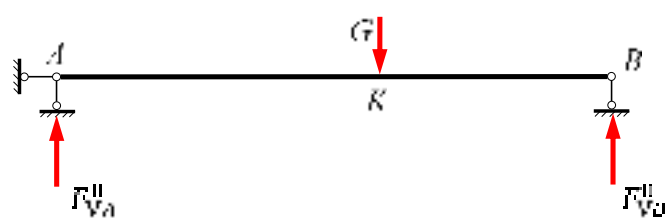

(b) Simply-supported-beam of concentrated load

Figure 4. Schematic of delivery stage 
Characters in Figure 4 and their meanings are explained as below:

- $F_{\mathrm{RA}}^{\prime}, F_{\mathrm{VA}}^{\prime}$ : component forces in the vertical direction and the chord $A B$ direction of tension of sending extremity $F_{\mathrm{T} A}$

- $F_{\mathrm{R} B}^{\prime}, F_{\mathrm{V} B}^{\prime}$ : component forces in the vertical direction and the chord $A B$ direction of tension of sending extremity $F_{\mathrm{T} B}$

- $F_{\mathrm{VA}}^{0}, F_{\mathrm{V} B}^{0}$ : counter-forces of supports $(A$ and $B)$ of simply-supported-beam.

- $K$ : action point of concentrated load $G$.

- $f_{K}$ : vertical distance between point $K$ and chord $A B$.

In a balanced situation, relationships of forces in Figure 4 are shown in (23):

$$
\left\{\begin{array}{l}
F_{\mathrm{V} A}^{\prime}=F_{\mathrm{V} A}^{0} \\
F_{\mathrm{V} B}^{\prime}=F_{\mathrm{V} B}^{0} \\
F_{\mathrm{R} A}^{\prime}=F_{\mathrm{R} B}^{\prime}
\end{array}\right.
$$

Then, decomposing $F_{\mathrm{R} A}^{\prime}$ along horizontal and vertical directions respectively will get $F_{\mathrm{H}}$ and $F_{\mathrm{H}} \tan \theta$. Similar to previous stages, there is no load exerted on highline cable in the horizontal direction. Thus, horizontal components of tension of all the points on the highline cable are always $F_{\mathrm{H}}$. Thus, $F_{\mathrm{R} B}^{\prime}$ can also be decomposed into $F_{\mathrm{H}}$ and $F_{\mathrm{H}} \tan \theta$. Likewise, $F_{\mathrm{T} A}$ can be decomposed into $F_{\mathrm{H}}$ in the horizontal direction and $F_{\mathrm{V} A}$ in the vertical direction; $F_{\mathrm{T} B}$ can be decomposed into $F_{\mathrm{H}}$ in the horizontal direction and $F_{\mathrm{V} B}$ in the vertical direction. The relationship between these forces and forces in (23) is shown in (24).

$$
\left\{\begin{array}{l}
F_{\mathrm{H}}=F_{\mathrm{R} A}^{\prime} \cos \theta=F_{\mathrm{R} B}^{\prime} \cos \theta \\
F_{\mathrm{V} A}=F_{\mathrm{V} A}^{0}+F_{\mathrm{H}} \tan \theta \\
F_{\mathrm{V} B}=F_{\mathrm{V} B}^{0}-F_{\mathrm{H}} \tan \theta
\end{array}\right.
$$

Actually, given an action point $K$ of concentrated loads $G, F_{\mathrm{H}}$ can be determined by (25):

$$
F_{\mathrm{H}}=\frac{M_{K}^{0}}{f_{K}}
$$

$f_{K}$ in (25) can be computed by (26):

$$
f_{K}=\left|y_{K}-y_{B}\right|+\left|x_{K}-x_{B}\right| \tan \theta
$$

$M_{K}^{0}$ in (25) represents the bending moment of $K$ inference of a simply-supported-beam, which can be calculated using (27):

$$
M_{K}^{0}=F_{\mathrm{V} B}^{0}\left|x_{K}-x_{B}\right|
$$

Then, deduce the only unknown $F_{\mathrm{V} B}^{0}$ in (27). Because the bending moment equals the algebraic sum of all moments about the centroid of cross section caused by external forces on the side of the cross section and $\sum M_{A}=0$ in balanced situation, (28) can be obtained.

$$
\sum M_{A}=F_{\mathrm{V} B}^{0}\left|x_{A}-x_{B}\right|-G\left|x_{A}-x_{K}\right|=0
$$

On the basis of (28), the expression of $F_{\mathrm{V} B}^{0}$ represented in (29) can be deduced: 


$$
F_{\mathrm{V} B}^{0}=\frac{G\left|x_{A}-x_{K}\right|}{\left|x_{A}-x_{B}\right|}
$$

The value of $M_{K}^{0}$ can be obtained by plugging the result of (29) into (27). Then, put the results of (26) and (27) into (25), and the value of $F_{\mathrm{H}}$ will be obtained.

Additionally, there is a relationship according to Figure 4(b):

$$
F_{\mathrm{V} A}^{0}=G-F_{\mathrm{V} B}^{0}
$$

After calculations of (24), (25), (29), and (30), all the parameters that are needed to compute $F_{\mathrm{VA}}$ and $F_{\mathrm{V} B}$ are known. Finally, $F_{\mathrm{TA}}$ and $F_{\mathrm{T} B}$ can be obtained using the parallelogram law, as (31) shows:

$$
\left\{\begin{array}{l}
F_{\mathrm{TA}}=\sqrt{F_{\mathrm{VA}}^{2}+F_{\mathrm{H}}^{2}} \\
F_{\mathrm{T} B}=\sqrt{F_{\mathrm{V} B}^{2}+F_{\mathrm{H}}^{2}}
\end{array}\right.
$$

Furthermore, given coordinates of points $A, K$, and $B$, plugging the coordinates of neighboring two points into the twopoint form of a straight-line will achieve the shape equation of this cable segment. Simultaneous equations of these equations are the shape equation of the whole highline cable:

$$
\left\{\begin{array}{l}
\frac{x-x_{A}}{x_{K}-x_{A}}=\frac{y-y_{A}}{y_{K}-y_{A}}, x_{A} \leq x<x_{K} \\
\frac{x-x_{K}}{x_{B}-x_{K}}=\frac{y-y_{K}}{y_{B}-y_{K}}, x_{K} \leq x \leq x_{B}
\end{array}\right.
$$

Plugging the coordinates of neighboring two points into the Euclidean distance formula will calculate the length of this cable segment, the sum of which is the total length of highline cable:

$$
S=\sqrt{\left(x_{K}-x_{A}\right)^{2}+\left(y_{K}-y_{A}\right)^{2}}+\sqrt{\left(x_{B}-x_{K}\right)^{2}+\left(y_{B}-y_{K}\right)^{2}}
$$

\section{Process and Result of Modeling}

\subsection{Introduction of Modeling Platform}

The modeling platform is Unity software, whose version is Unity 5.6.2. Requisite scripts of algorithmic sub models and physical sub models are written in C\# using MonoDevelop editor, which comes with Unity software.

The physical sub model can be divided into two parts, that is, scene and display. Part of the display consists of several graphical user interfaces (GUI). Part of the scene is constituted by many physical objects, such as highline cable objects, replenishing vessel object, receiving vessel object, replenishment equipment objects (e.g., replenishing pillar and receiving pillar) cargo object, and environment objects (e.g. sea and sky). All of these objects are created on the basis of different plugins of Unity software and are designed and organized according to the real replenishment situation. Highline cable objects that are important in physical sub models are attached to certain modeling scripts or have parameters set according to the requirement of each replenishment stage and the results of the algorithmic sub model.

\subsection{Values of Important Parameters}

Global parameters: The vertical distance between the sending extremity and receiving extremity is marked as $c$ and is set as $16 \mathrm{~m}$. The horizontal distance between the sending extremity and receiving extremity is marked as $l$ and is set as $60 \mathrm{~m}$. 
Local parameters: The distributed load $q$ is set as $40 \mathrm{~N} / \mathrm{m}$. The constant tension of the sending extremity at the adjustment stage and unloading stage is marked as $F_{\mathrm{TA}}$ and is set as $80000 \mathrm{~N}$. The gravity of one cargo $G$ is $20000 \mathrm{~N}$.

5.3. Results and Analyses of Simulation Models

Screenshots of simulation models built in this paper are shown in Figure 5. Because the model of the erection stage and the model of the withdrawal stage are essentially the same, we have only shown the model of the erection stage.

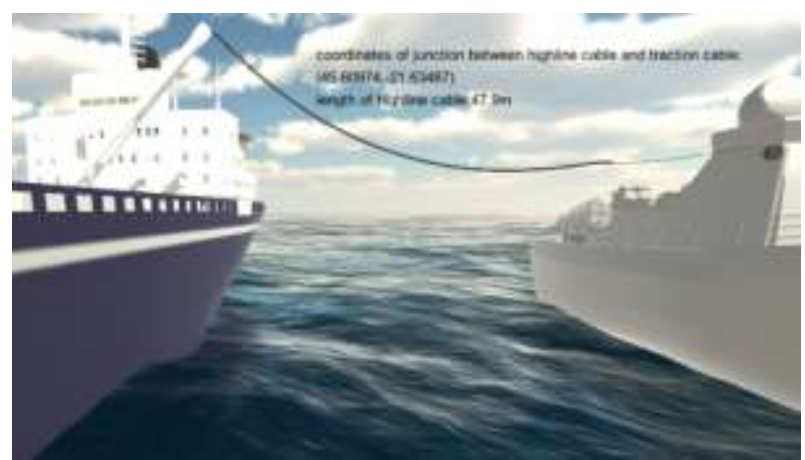

(a) Erection stage

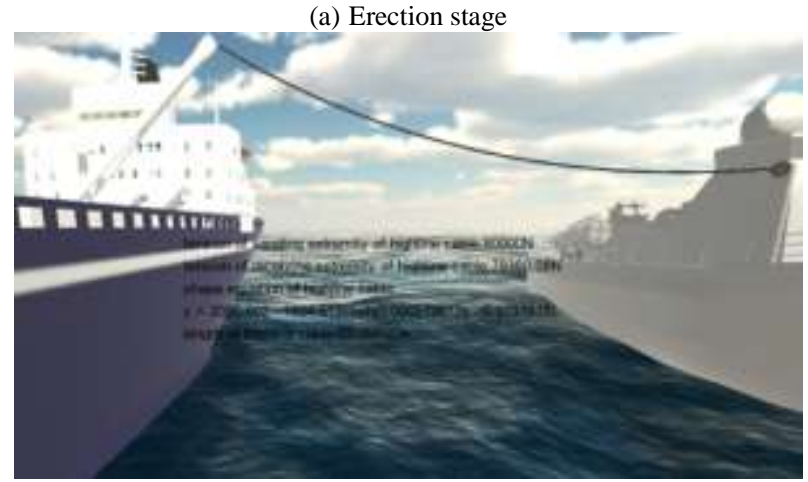

(c) Adjustment stage

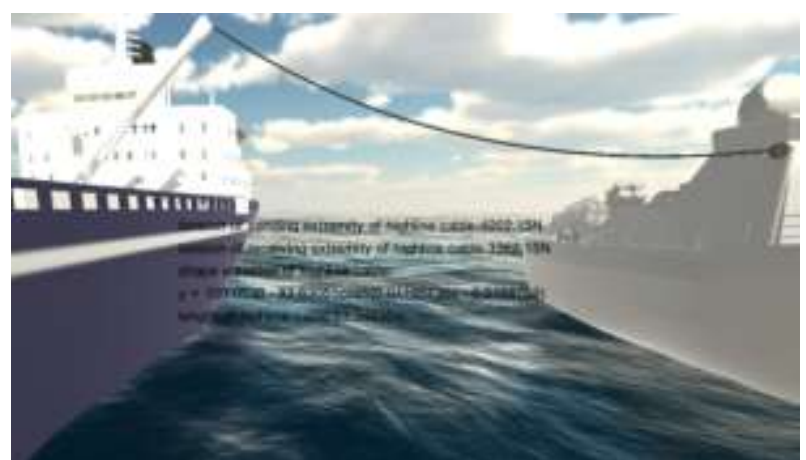

(b) Stabilization stage

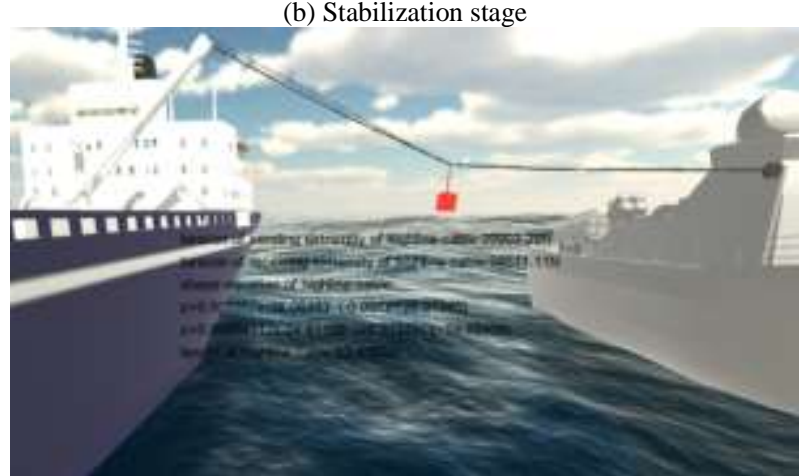

(d) Delivery stage

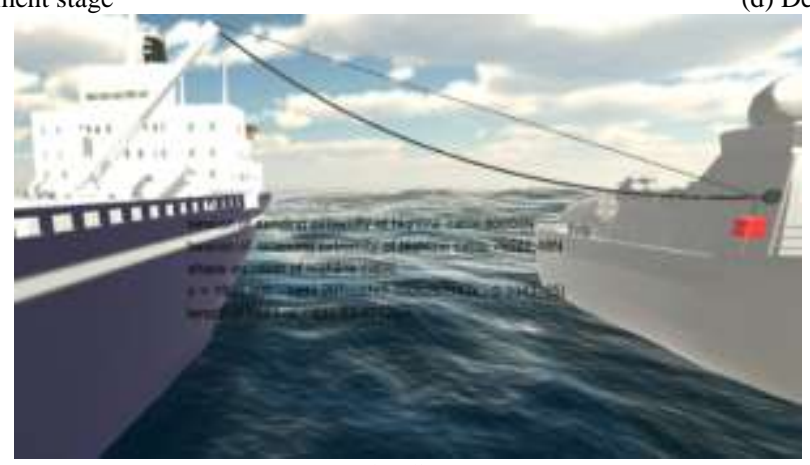

(e) Unloading stage

Figure 5. Screenshots of simulation models

In Section 3.2, two kinds of iterative methods are designed to solve equations of highline cable in different conditions. Results of these two methods are shown in Table 1, and they are nearly the same.

\begin{tabular}{|c|c|c|c|c|c|}
\multicolumn{7}{|c|}{ Table 1. Results of two iterative methods } \\
Known Parameters & $S$ & $f$ & $F_{\mathrm{T} A}$ & $F_{\mathrm{T} B}$ & $\beta$ \\
\hline$f$ & & & & & \\
\hline$S$ & 63.34896 & $\mathbf{5 . 6 2 0 9 9 9}$ & 4002.150 & 3362.150 & 0.3587208 \\
\hline & $\mathbf{6 3 . 3 4 8 9 6}$ & 5.620999 & 4002.149 & 3362.149 & 0.3587209 \\
\hline
\end{tabular}


Results of the iterative method in Section 3.3 tells us that $F_{\mathrm{T} B}=79360.08 \mathrm{~N}$. Hence, $\Delta$, the percentage of difference between the tensions of two extremities equals $\left|\frac{F_{\mathrm{T} B}-F_{\mathrm{TA}}}{F_{\mathrm{T} B}}\right|=0.80635 \%$, which satisfies the rule in [11] that $\Delta \leq 10 \%$.

\section{Conclusions}

This paper develops a kind of simulation model to reflect how the shape of highline cable changes at various stages of alongside replenishment for solid cargo. The model contains the algorithmic sub model and the physical sub model. The algorithmic sub model of different stages is based on forced situations of highline cable. At stages when highline cable is under the action of distributed load, the theory of catenary is used to deduce the shape equation of highline cable. Meanwhile, at stages when highline cable is under the action of concentrated load, the theory of structural mechanics is used to make such deductions. Different iterative methods are designed to solve equations made by the theory of catenary in specific situations. The physical sub model is established in Unity software based on actual situations and results of the algorithmic sub model. This simulation model is able to not only display how the shape of highline cable changes visually but also print values of these relevant physical properties on the screen in real time, which combines qualitative analysis and quantificational analysis. Results of execution indicate that the model can reliably mirror changes of highline cable at various stages of replenishment. Therefore, this model can be applied to assist in training operators of replenishment at sea.

\section{References}

1. D. P. Zhang, K. Q. Zhu, Y. Bai, J. Liu, and S. P. Wang, "Dynamic Analysis of Marine Alongside Replenishment for Highline Cable System," Ship Engineering, Vol. 39, No. 1, pp. 59-63, 2017

2. N. Li, Z. B. Wei, X. J. He, S. Y. Zhang, and A. D. Ren, "Multibody Dynamics Model of Highline Cable," Journal of Naval University of Engineering, Vol. 26, No. 6, pp. 27-31, 2014

3. N. Li, Z. B. Wei, X. J. He, S. Y. Zhang, and A. D. Ren, "Rigid-flexible Dynamic Model of Highline Cable," Journal of Naval University of Engineering, Vol. 28, No. 2, pp. 15-19, 2016

4. N. Li, Z. B. Wei, S. Y. Zhang, and A. D. Ren, "Modelling and Simulation of Highline Cable for Replenishment at Sea," Acta Armamentarii, Vol. 37, No. 4, pp. 735-743, 2016

5. N. Li, Z. B. Wei, X. J. He, and S. Y. Zhang, "Research on Highline Cable Parametric Design Platform of Alongside Replenishment at Sea," Journal of System Simulation, Vol. 28, No. 7, pp. 1481-1488, 2016

6. W. Zhao and Y. Wang, "Simulation of Alongside Solid Cargo Underway Replenishment System at Sea," Ship Engineering, Vol. 37, pp. 110-116, 2015

7. W. Zhao, H. K. Zhao, and Y. Wang, "Dynamic Performance Simulation of Underway Replenishment at Sea Highline Constant Tensile Control System,” Ship Engineering, Vol. 35, No. 2, pp. 55-58, 2013

8. R. G. Ma, L. X. Zhang, and X. J. He, "Analysis the Static Influence of Highline Cable Alongside Replenishment for Dry Cargo," Ship Science and Technology, Vol. 34, No. 2, pp. 47-49, 2012

9. K. Kamrin and L. Mahadevan, "Soft catenaries," Journal of Fluid Mechanics, Vol. 691, pp. 165-177, 2012

10. L. K. Li, "Structural Mechanics Book 2," 6th Edition, Higher Education Press, Beijing, 2017

11. A. D. Ren, D. W. Zhang, and X. J. He, "Constant Stress Consumption in the Research of Highline System," Ship Science and Technology, Vol. 32, No. 2, pp. 27-31, 2010 